BOOK REVIEWS

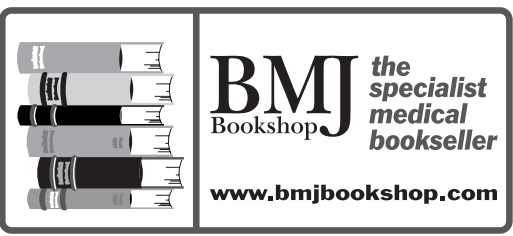

Toxicity and risk: context, principles and practice

Illing P. (Pp ix + 154; £21.99) 2001. London and New York: Taylor and Francis Books Ltd. ISBN 0415233712

“... round the rugged risks the wretched regulator ran ..." (with apologies to the old tongue twister!).

We live in an industrialised world, where many believe we enjoy chemical gain at the cost of societal pain. As a result, the prediction and evaluation of risk and the detection or exclusion of the harm caused, most often attributed to chemicals, but not excluding physical dangers, too, such as atomic energy and transport accidents, has become a majo industry in its own right. "Risk assessment" is a vital set of procedures for safeguarding the individual, the community and the environment, while still, we hope, permitting us to enjoy the benefits of various sources of risk There are many other ways in which chemical and physical risks may occur that can imperil the individual, society, and the environment, which are little regulated but which can still be evaluated by the same mixture of philosophical and quasi-scientific procedures as are applied to the better identified risks.

Professor Illing has written an effective short primer on the general nature of "risk" concentrated on toxicity as it may occur in industry, but not excluding medicines, domestic exposures, physical dangers, and other sources. He has provided a clear account of what such "risks" comprise, how the corresponding "hazards" may be identified, and how the resultant risks are dealt with, principally from a governmental viewpoint. He has not been afraid to bring out many of the associated problems of "risk management", notably the essential but awkward and often covert attempt at "risk-benefit" analysis, and the consequential difficulties of "risk communication". He shows how the former forces the risk manager to find some metric common to the risk and the benefit, so that a temperate judgement can be made of their relative worth, followed by some means of "communication" - that is, of selling the proposed solution to those affected, such as an industry which wishes to sell products or cause pollution, and workers and other voters who may have to put up with it. Political considerations form an increasing element of risk assessment and management, and although this book is written in a detached and reasonably academic fashion, the reader can never afford to forget that real or imaginary concerns about "risks" are a potent source of political agitation and unbalanced societal decision making.

The book provides a workmanlike account of the procedures used by many in these areas, paying attention mostly to scientific aspects, and it illustrates at length many of the official organisations and procedures adopted nationally, in the European Union, and in a variety of international organisations. It does not present many new examples, not even from the field of major industrial hazards, a particular professional interest of the author, but it does provide a clear account of principles and problems, and is well illustrated with diagrams, tables, and various pictograms, which illustrate procedures employed and factors that should be considered. Care has been taken to present clear definitions and straightforward definitions. There are seven pages of up to date references, drawn from British, American, and international literature, and a helpful level of indexing.

This book is a helpful, clear, and useful account of "risk". It will be of equal value to students and practitioners of risk analysis, as a timely account of current ideas, and it would be of no less assistance to organisational and self appointed agitators and spokespersons, who would be helped to understand more about the position of their concerns in modern society and both how to communicate with and to understand communications from officialdom.

A Dayan

\section{Language and the Internet}

Crystal D. (Pp 272; £13.95, \$19.95) 2001. Cambridge: Cambridge University Press. ISBN 0521802121 (hardback)

Do you txt? Have you ever used a smiley :) ? Do you know what wtfigo means? Do you wince — even just a little —at the greengrocer's cavalier use of the apostrophe? If you answered yes to these questions, then you should read this book. If you answered no to any of them, then you need to read this book, because something is happening to the English language, and you need to know what it is. David Crystal is a noted linguist who, for the first time, has attempted to analyse the language of the Internet and the World Wide Web. Crystal has given this form of language the Orwellian name Netspeak, and has analysed its use in e-mails, in chat groups, in virtual worlds, and on the Web. He has sought to determine whether or not Netspeak has the attributes of a distinct language, but concludes that while it is a genuine third medium, distinct from speech and writing, it is more a dialect of English than anything else.

This conclusion is not arrived at via a totally dry linguistic analysis. Crystal does provide an interesting discussion on the "prescriptive" approach to language (typified by the view that a split infinitive represents the end of civilisation as we know it) versus the "descriptive" liberal approach, which views widely used deviations from the "norm" as evidence of a healthy evolution and growth of a language. Crystal clearly sets himself in the latter camp as far as "Netspeak" is concerned, although he does make the point that a very widely used word processing software package automatically warns, and potentially stifles individualism in, authors when they stray outside what could be considered arbitrary grammatical rules. (Just try your spell check routine.)

Although there is a short discussion of the phenomenal rise in mobile telephone use and the implications for the language through the increasing use of text messages, the book was finished before perhaps the full significance of this development could be analysed and assessed. But this is inevitable in any book which deals with digital technology-as the author himself points out, such an exercise will inevitably be out of date in some respect before it is published. A second edition could be expected to address this issue in more depth.

There is a fascinating section on the question of whether or not the globalisation of the Internet and World Wide Web will lead to a worldwide domination of English as the lingua franca-perhaps that should be lingua electronica-of the Internet. I have to declare an interest, coming from the Celtic fringe, but this is not the first time in history that the prospect of a hegemony of English has been in prospect. Crystal argues that far from it, the Internet provides an even greater opportunity for lesser used languages to prosper and thrive. Time will tell.

Crystal has written a stimulating book, and he has done this largely in layman's terms, although there are occasional lapses into phrases like "hyperverbal sonic prominence", and for a linguist to start a sentence with "Other factors than ..." jars more than a little. However, if you have an interest in language and use a computer, you should read this book. Using the extensive tables of acronyms for useful (and occasionally rude) phrases you can also impress your friends and e-correspondents; you could even construct sentences without the use of a single "proper" English word if you tried. Cul8r

M Williams

\section{Antidotes}

Edited by: Flanagan and Jones (Pp 325; $£ 39.99 \mid 2001$. Andover: Taylor \& Francis. ISBN 0748409653

This very welcome book on antidotes by Flanagan and Jones from Guy's and St Thomas' NHS Trust is a paperback, but fairly comprehensive text, on antidotes. It includes a chapter on chemical warfare agents by myself and Bob Maynard, on which I will not comment, except to say that, sadly, this chapter may prove a selling point at this time.

Chapter 1 is a general introduction, with fascinating sections on the mechanism of action of antidotes and their history and development from ancient times. I had not realised how much time nineteenth century doctors spent on painters' colic (lead poisoning). Also in this chapter is a list of obsolete antidotes.

Thereafter the book assumes a more systematic character, starting with the treatment of metal poisoning. However, as shown by the section on lead poisoning, the book is not a 
simple compendium of antidotes (as the title suggests) and the treatment of the various types of lead intoxication, including low level exposure, is discussed. The next two chapters discuss, respectively, immunotherapy and metabolic antidotes. Chapter 5 (pharmacological antidotes) covers the various antidotes acting at characterised receptors and includes discussion of the oxime cholinesterase reactivators. Chapter 6 comprises an interesting discussion of carbon monoxide, cyanide, and hydrogen sulphide antidotes; the number of cyanide antidotes around the world attests to the lack of agreed protocols for the comparison of antidotes. The last chapters deal with paraquat and interferences with toxicological analyses. The book is good value for money at $£ 40.00$.

This is not an encyclopaedic book, but it is full of material for the clinician. Unusually, it is extremely chemically literate, with CAS numbers and chemical formulae. It will be invaluable to pharmacologists, health professionals interested in toxicology, and occupational health physicians.

T Marrs

\section{Community emergency preparedness: a manual for managers and policy makers}

World Health Organisation. (Pp 141; Sw Fr 42). 1999. Geneva: World Health Organisation. ISBN 9241545194

Human and economic losses from natural disasters continue to rise around the world, a trend punctuated by highly devastating events such as Hurricane Mitch in Central America (1998) and the earthquake in Gujarat in India in 2000, when tens of thousands of people were killed or severely injured and communities containing hundreds of thousands of people have still not recovered from the inflicted damage. The International Decade for Natural Disaster Reduction (IDNDR) was launched by the United Nations in the 1990s, in a bold attempt to reduce such losses by transferring knowledge to mitigate hazards to the developing world where $90 \%$ of natural disasters and $96 \%$ of the deaths occur The IDNDR did have the effect of focusing the attention of many scientists, especially those involved in the earth and meteorological sciences and engineering, towards the application of their work in reducing natural hazards. The concepts of mitigation, or disaster prevention, also spread to a much wider audience, including national and local government policy makers charged with emergency management. This book is timely in summarising disaster reduction in the terms of emergency preparedness and in a way that can be used to support the development of policies and enlisting the involvement of a wide range of local and national agencies. It might therefore be expected to be useful reading for an even wider audience, such as health practitioners in occupational and environmental health, public health workers, and groups involved in emergency response. Disaster planning is slowly moving up the agenda of global public health, partly because of growing awareness that climate change will worsen the risk of floods and windstorms in many countries, but also because they are "unexpected events", in the same bracket as emergent diseases and biochemical terrorism incidents. Parallels are also seen in industry, such as the Bhopal chemical release in India in 1984, and the undermining of consumer confidence in Belgium in 1999 when dairy products became contaminated with dioxin These and similar toxic incidents have wide industrial and political reverberations, making corporations extend their risk management strategies from occupational hazards to a wider environmental remit. This is not all: business is now increasingly being perceived as a process of risk management in which "salient events" can provoke sudden, seismic shifts in the financial and customer markets that threaten the viability of corporations and even governments.

Unfortunately, however, this book has overshot the zeitgeist in its attempts to unify the disaster field by generalising its approach to what are quite different phenomena. Thus the impacts and planning issues for floods are very different from chemical releases, for example. The authors have obviously been attracted to applying the language of mitigation across the board, while ignoring the large practical differences of dealing with the separate hazards. This superficial approach seriously underestimates the scope of natural and technological disaster reduction and hence the measures needed for coping with catastrophe. The book does make a laudable attempt to put the different disaster impacts into risk scales and scoring systems, and these will at least make the reader reflect on the way in which scientific advances in understanding extreme natural processes can illuminate what for many is an arcane subject. Indeed, computer modelling of natural processes, including meteorological and climate phenomena, are starting to drive our understanding of the ways in which the natural world behaves and causes damage, together with loss of life, in extreme events. Since the 1980s it has been possible to model technological hazards, such as the dispersion of dense gases and the effects of explosions of flammable substances, a development that has enabled major incident planning for chemical hazards to be a regulatory requirement. We are on the verge of similar breakthroughs with floods and volcanic eruptions using numerical simulation on a more scientific footing. By analogy, world financial markets cannot run their risks without mathematical models to help reduce uncertainty to manageable levels. But learning to come to terms with scientific uncertainty is an essential prerequisite of disaster mitigation work and no manager should set about emergency preparedness unless he or she understands this. Unfortunately this book shows little awareness of the scientific tools needed for the job in hand and their limitations. Who plans for a publication devoted entirely to health sector preparedness should include drawing on a wider diversity of interdisciplinary expertise than is evident in this volume.

P J Baxter 